\title{
Las Tecnologías de la Información y Comunicación y el Diseño de Servicios en Cuba: un estudio de caso sobre el Paquete Semanal
}

Information and Communication Technologies and Service Design in Cuba: a case study on the Weekly Package

As Tecnologias da Informação e Comunicação e o Design de Serviços en Cuba: um estudo de caso sobre o Pacote Semanal

DOI: $10.1590 / 1809-5844201928$

\section{Yanet Castellanos Argüelles ${ }^{1}$}

https://orcid.org/0000-0002-7814-1853

Ana Carolina Kalume Maranhão ${ }^{2}$

https://orcid.org/0000-0002-5321-9191

\section{Rogerio José Câmara ${ }^{1}$}

https://orcid.org/0000-0002-1590-4948

${ }^{1}$ (Universidade de Brasília, Instituto de Artes, Programa de Pós-Graduação em Design. Brasília - DF, Brasil).

2(Universidade de Brasília, Faculdade de Comunicação, Departamento de Jornalismo. Brasília - DF, Brasil. Universidade de Brasília, Instituto de Artes, Programa de Pós-Graduação em Design. Brasília - DF, Brasil).

\section{Resumen}

En Cuba, devido a limitaciones de acceso a Internet, la población creó una solución innovadora para obtener informaciones disponibles en las redes, llamada de Paquete Semanal (PS), un compendio de informaciones que integra diferentes contenidos digitales, extraídos de la Internet y comercializados en discos duros y memorias flash. La venta del PS se convirtió en negocio y generó la competencia entre los vendedores, configurándose como diseño de servicios organizado de modo espontáneo, surgido de una energencia de la necesidad de consumo de información, asimilada por el mercado negro. Este trabajo busca comprender el flujo de circulación del PS vinculando análisis cualitativo y cuantitativo, para identificar los principales actores, así como las preferencias informacionales de los usuarios, en la ciudad de Santa Clara, en Cuba. Frente a la restricción de acceso a la información en el país, el PS se presenta como um recurso com vasta aceptación y usabilidad entre los cubanos. Palabras clave: Cuba. Investigación cualitativa y cuantitativa. Producción de Contenido Digital. Tecnologias de la Información y Comunicación. Diseño de servicios. 


\begin{abstract}
In Cuba, due to the limitations of Internet access, the population created innovative solutions to obtain information available on the networks, called the Weekly Package (PS), a compendium of information that integrates different digital content, extracted from the Internet and marketed on hard drives and flash menories. The sale of the PS became a business and generated competition among sellers, configuring itself as service design organized spontaneously, arising from an emergency of the need for information consumption, assimilated by the black market. This work seeks to understand the flow of PS circulation by linking qualitative and quantitative analysis, to identify the main actors, as well as the informational preferences of the users, in the city of Santa Clara, in Cuba. Faced with the restriction of access to information in the country, the PS presents itself as a resource with great acceptance and usability among Cubans.
\end{abstract}

Keywords: Cuban. Qualitative and Quantitative Research. Digital Content Production. Information and Communication Technologies. Service design.

\title{
Resumo
}

Em Cuba, devido às limitações de acesso à Internet, a população criou uma solução inovadora para obter informações disponíveis nas redes, denominada Pacote Semanal (PS), um compêndio de informações que integra diferentes conteúdos digitais, extraídos da Internet e comercializados en HDs e pendrives. A venda do PS tornou-se um negócio e gerou concorrência entre os vendedores, configurando-se como design de serviços organizado de modo espontâneo, surgido de uma emergência sobre a necessidade de consumo de informação, assimilada pelo mercado negro. Este trabalho busca compreender o fluxo de circulação do PS, vinculando análises qualitativas e quantitativas, para identificar os principais atores, bem como as preferências informacionais dos usuários, na cidade de Santa Clara, en Cuba. Diante da restrição de acesso à informação no país, o PS apresenta-se como um recurso com grande aceitação e usabilidade entre os cubanos.

Palavras-chave: Cuba. Pesquisa Qualitativa e Quantitativa. Produção de Conteúdo Digital. Tecnologias da Informação e Comunicação. Design de serviços.

\section{Introducción}

En Cuba, el acceso a Internet es restringido por la baja conectividad, precios elevados y proporcionada por una única enpresa de telecomunicaciones en el país, una enpresa pública ${ }^{1}$ que responde al estado, siendo esta la Enpresa de Telecomunicaciones de Cuba Sociedad Anónima, ETECSA (MINCOM, 2018). En general, la población no tiene conexión en casa y, mucho menos, a movilidad de acceso vía smartphones. Actualmente, el precio de una hora de acceso a Internet cuesta aproximadamente o 4,3 \% del salario medio nacional, que es de \$ 584,00 pesos cubanos (23 CUC), (ETECSA, [S.d.]; MINISTERIO DE JUSTICIA, 2005), equivalente a \$23,00 USD (United States Dollar), conforme Banco Central de Cuba

1 En Cuba todas las enpresas son públicas, o sea, pertenecen al gobierno. (CONSTITUCIÓN DE LA REPÚBLICA DE CUBA, 1976). 
(2016) y Banco Metropolitano de Cuba (2016), esto significa aproximadamente 24h de conexión a Internet, si todo el salario medio recibido fuera gastado en su consumo. Además, la Internet tiene áreas específicas en las ciudades de Cuba (las que se han ido increnentando paulatinamente), en las cuales es posible conectarse por medio de una señal wifi local, no obstante, la velocidad de la red es lenta.

Cuba enfrenta dificultades políticas y socioeconómicas desde 1959. Con la implantación del socialismo, el país se vio en medio de conflictos históricos, sobre todo con los Estados Unidos, sufriendo diversos embargos. Situación que limita el desarrollo tecnológico y el acceso a los medios de comunicación como la Internet, reduciendo las posibilidades de troca de informaciones con el mundo. Se compromete así la percepción del mundo sobre la isla y resta a la población cubana una información mediada por periódicos nacionales y cinco canales de televisión educativos, los que incluyen programas de entretenimientos.

Con las dificultades de acceso a las informaciones disponibles en Internet, la población cubana ha creado formas alternativas de acceso a ellas, tales como el uso de menorias flash y discos duros como medio de obtención y compartimiento. El método más utilizado en la Cuba de hoy se denomina "Paquete Semanal" (PS), un conjunto de datos digitales, comercializados senanalmente junto a la población en menorias flash y discos duros.

De acuerdo con Concepción, (2016a), el Paquete Senanal "surge como una alternativa para las circunstancias desfavorables de dos medios: televisión e Internet” y representa "un compendio de materiales que abarca todos los tipos de propuestas, desde los más variados géneros televisivos y cinenatográficos hasta aplicaciones informáticas" (CONCEPCIÓN, 2016b).

La distribución del PS, como el nombre lo indica, es senanal. El contenido no sufre muchas variaciones de una senana para outra. Se busca dar continuidad a los contenidos audiovisuales publicados en el paquete de la senana anterior, así como actualizaciones de software y antivirus. La cantidad de información comprendida en este conjunto alcanza hasta un terabyte de capacidad.

Las informaciones del paquete resultan del trabajo de un equipo llamado Matriz del Paquete Semanal, con acceso a Internet y varios equipamientos tecnológicos que les permiten descargar las informaciones. Resultan también de la realización de copias de vídeos a través de antenas parabólicas de televisión ${ }^{2}$, adenás de producciones independientes que encuentran en el paquete senanal un mercado. Estos paquetes semanales y sus modos de circulación son objetos del presente artículo, desarrollado desde la perspectiva del diseño de servicio ${ }^{3}$, a partir de investigaciones realizadas en el Consejo Popular "Condado Norte”, ubicado en

2 Información obtenida a partir de entrevista realizada a uno de los creadores del paquete senanal.

3 Según Pinheiro (2015), el diseño de servicios “mezcla las perspectivas humanísticas del diseño con la lógica orientada a servicio, con el objetivo de proporcionar una forma enocional y centrada en el ser humano que los lleve a crear nuevos servicios o mejorar los ya existente. Pinheiro (2015) afirma que aplicar el diseño de servicios no deve ser una práctica especializada ni una disciplina académica, pero si reconocida como una habilidad, aliada a la competencia y actitud, lo que puede ser aprendido y aplicado en diferentes profesiones, objetivos y áreas del conocimiento”. 
el municipio de Santa Clara, capital de la provincia Villa Clara, Cuba, en el centro del país. El Consejo Popular es el órgano más directamente ligado a los ciudadanos y a los centros laborales; actúa en un determinado territorio y apoya los órganos del Poder Popular municipal, provincial y nacional (CONSEJO POPULAR, [S.d.]).

Partiendo del contexto presentado, este artículo tiene como objetivo analizar los canales de distribución y las preferencias en lo que se refiere a contenidos digitales, de los consumidores del paquete senanal en la ciudad de Santa Clara, a partir de una investigación de métodos mixtos, involucrando análisis cualitativos y cuantitativos y la metodología básica del diseño de servicios, para entender el funcionamiento de este fenómeno nacional, en Cuba.

\section{Procedimientos metodológicos}

Para la realización de esta investigación, fue elaborado un estudio que relaciona la investigación cualitativa y la cuantitativa en diferentes fases del proceso, con vistas a un estudio de caso, que realiza la recopilación de datos a través de la observación participante, entrevistas en profundidad y aplicación de cuestionarios. Para la aplicación de estos instrumentos, así como la realización de los análisis y discusión de los resultados, se siguió el camino metodológico propuesto por Maranhão (2017), el cual se presenta en la Figura 1, a continuación.

Figura 1 - Pasos metodológicos seguidos en la investigación

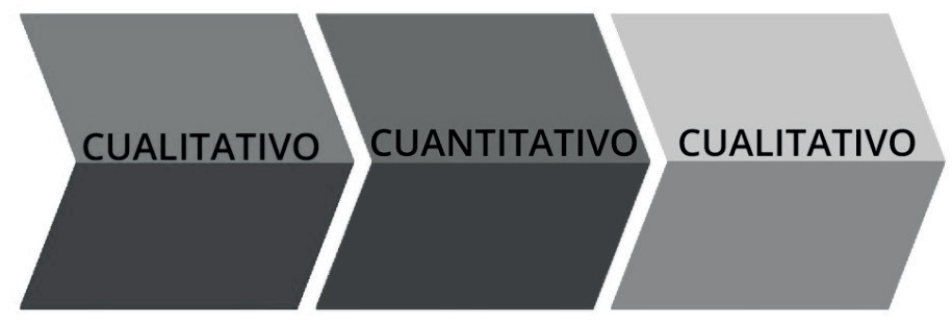

Fuente: Modificado de Maranhão (2017).

La investigación fue concebida entres fases, como se muestra en la Figura 1, siendo que la primera fase está compuesta por el enfoque metodológico cualitativo e incluye la definición de la unidad de caso, elaboración y aplicación de los instrumentos de la recopilación de datos y aplicación de tres de las diversas herramientas del diseño de servicio ${ }^{4}$ como será presentado más adelante. En la segunda fase, se enpleó el enfoque metodológico cuantitativo, que consistió en la aplicación y análisis estadístico de los cuestionarios. En

4 El diseño de servicios comprende un conjunto de herramientas asociados a las etapas de exploración, creación, reflexión e implenentación. En este artículo fueron usadas herramientas como el mapa de stakeholders, entrevistas contextuales y la co-creación. 
la última fase se realizaron análisis cualitativos de los resultados. Estas fases indican el proceso de concepción y ejecución de la investigación, las cuales serán detalladas en los siguientes itens.

\section{Selección de la muestra}

En la primera fase, fue mapeado el público meta por medio de la observación participante, con el objetivo de seleccionar la población que sería investigada en la ciudad de Santa Clara. Como recorte, entre los 19 Consejos Populares del Municipio, fue elegido el Consejo Popular "Condado Norte", por ser un área popularmente conocida como la más comercial de la ciudad, tanto de negocios lícitos como ilícitos. También por medio de la observación y el sondeo de la comunidad, fue posible detectar cinco vendedores del PS con los que se realizaron entrevistas en profundidad combinada con entrevistas contextuales, para identificar qué actores estaban involucrados en el proceso de producción y comercialización del paquete semanal. De las entrevistas con los vendedores, fueron detectados tres paqueteros 5 . Adenás, en esta fase, fue descubierto que los creadores del PS viven en la Habana, capital del país. Los métodos de recopilación de información y las herramientas del diseño de servicios fueron aplicados en una muestra escogida por conveniencia, dado la dificultad de acceder a todo el universo de clientes que consumen los productos comercializados en el PS.

Esta muestra está compuesta por: dos creadores del paquete semanal, tres paqueteros y cinco vendedores, sumando al contexto de la investigación 50 clientes-usuarios del paquete semanal. Estos clientes-usuarios fueron seleccionados considerando diez clientes-usuarios de cada uno de los cinco vendedores entrevistados, conforme presenta la Figura 2.

5 Son los responsables por la modificación y organización de las informaciones del PS en cada ciudad, para posterior traspaso y venta a los usuarios. 
Figura 2 - Muestra del estudio de caso

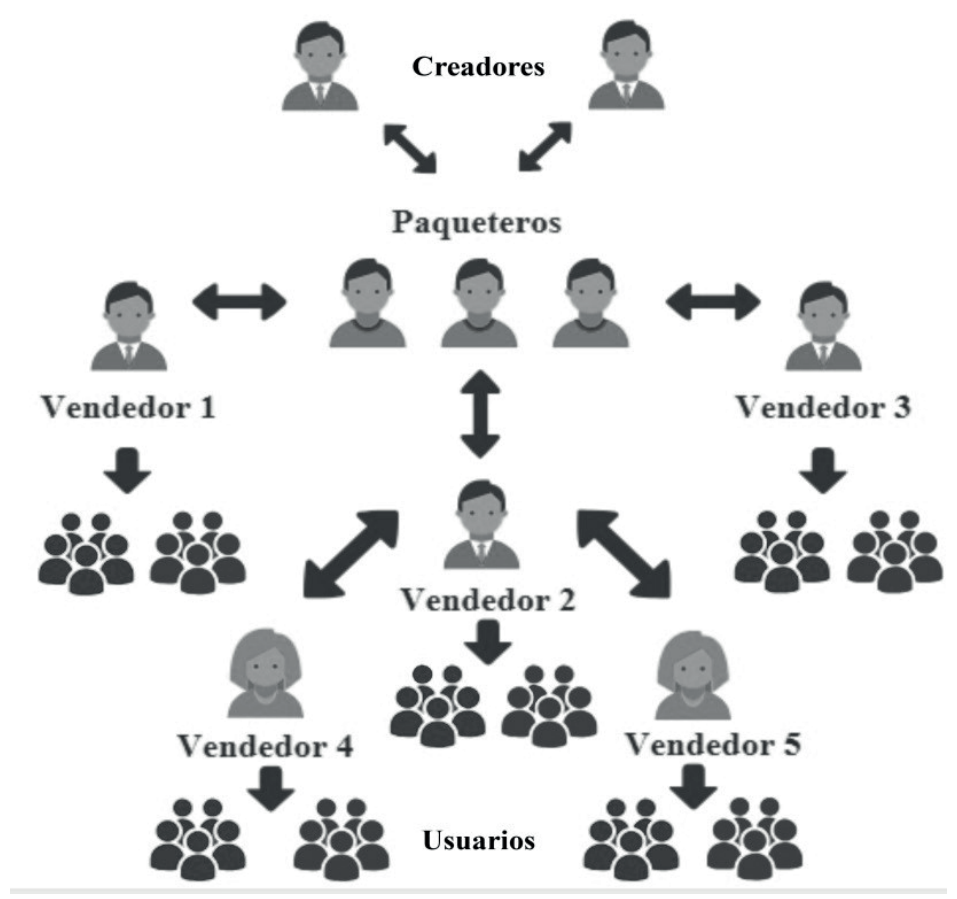

Fuente: Elaboración propia, jun. 2018.

Todas las preguntas para la recopilación de datos se elaboraron según la metodología de los procesos básicos del diseño de servicios, expuestos en "This is service design thinking” (STICKDORN; SCHNEIDER, 2011). Esta metodología se compone de cuatro etapas: exploración, creación, reflexión e implenentación, que permean las muchas fases de la investigación, dado que cada una de las etapas propone aspectos a medir, partiendo de las cuales se han concebido las preguntas de los instrumentos para la recopilación de datos.

Siguiendo a Stickdorn y Schneider (2011), la metodología básica del diseño de servicio se presenta como una caja de herramientas. Para la presente investigación, se enplearon tres herramientas del proceso básico del diseño de servicios, correspondientes a la etapa de exploración, fueron usadas el mapa de stakeholders y las entrevistas contextuales, y de las etapas de creación y reflexión la co-creación.

El mapa de los stakeholders es una representación visual o física de los públicos de determinado servicio. Para hacerlo, es necesario realizar una lista completa de las necesidades de los stakeholders, adenás de aplicar entrevistas. Es importante que en este proceso sean revelados los intereses y motivaciones de cada grupo y estos deben incorporarse también al mapa. Una vez que la lista esté completa, se debe enfocar en como esos grupos se interrelacionan e interactúan entre sí (STICKDORN; SCHNEIDER, 2011).

Una herramienta útil para la elaboración del mapa de stakeholders sería la entrevista contextual que, de acuerdo con Stickdorn e Schneider (2014), es desarrollada y conducida 
en el contexto donde se produce el servicio. Es una técnica etnográfica que permite que los entrevistadores observen el comportamiento en el cual están interesados. En cuanto la co-creación puede ser usada en combinación con outras herramientas del proceso de diseño de servicio. La sesión de co-creación, objetiva explorar vías posibles y recoger amplia variedad de perspectivas dentro del proceso de diseño de servicio.

De esta forma, el mapa de stakeholders fue utilizado para la identificación de todas las partes implicadas en el paquete senanal y las entrevistas contextuales con la intención de ayudar al entrevistado a recordar detalles específicos de lo que se está investigando. A la misma vez que los principios de la co-creación fueron enpleados para interpretar la composición de los CDs del PS, y los procesos de prestación del servicio de compra-venta.

En la segunda fase, se aplicaron los instrumentos investigativos a los 50 clientes/ usuarios de la muestra seleccionada. El formulario utilizado en las encuestas fue compuesto por 12 preguntas referentes al acceso y uso del paquete semanal de contenidos digitales. Tres de las preguntas del cuestionario (preguntas 1, 11 y 12) tenían la posibilidad de respuesta abierta. La aplicación de este método investigativo fue realizada entre los días del 16 al 26 de enero de 2017. Otro dato interesante es que las encuestas fueron anónimas, para que los encuestados se sintieran libres para escribir con más transparencia.

Las análisis de los interrogantes fueron cuantitativas univariadas, a partir de una base de datos que permitió la visualización de las respuestas obtenidas y mediante la elaboración de gráficos y tablas, con resultados cuantitativos de las variables del cuestionario. Después del análisis cuantitativo, se realizaron análisis descriptivos de las respuestas. La realización de la base de datos y los análisis descriptivos se realizaron a través de Microsoft Excel 2013.

Por último, en la tercera y última fase de la investigación, se realizó la evaluación de los resultados obtenidos, de forma descriptiva. Para ello se siguieron tres pasos: reducción de la información, disposición y transformación de la información e interpretación de la información (SAMPIERI; COLLADO; LUCIO, 2014). Se procuró abreviar la cantidad de información resultante de la recopilación de información para facilitar el análisis comprensivo y relevante, incluyendo la separación, clasificación y síntesis de toda la información. En consecuencia, se organizaron los datos para que el procesamiento fuera viable.

\section{Discusión y Análisis de los resultados}

La observación participante permitió identificar quienes eran los vendedores del paquete senanal y de los contenidos digitales. Es posible afirmar que cada tres cuadras existen vendedores, conocidos popularmente en español como llenadores de menorias. Estos vendedores ejercen la actividad como negocio particular y no existe ningún estándar geográfico para la localización de los mismos, ni un perfil establecido, pues existen vendedores que, adenás del negocio del paquete, presentan un vínculo laboral con el gobierno, y vendedores que sólo se dedican a este tipo de actividad. Fue posible detectar 
también quienes son los clientes/usuarios de cada vendedor, determinando que los clientes escogen a sus proveedores por la calidad del servicio recibido y no por la proximidad. No todos los usuarios del PS son precisamente clientes, es decir, la mayoría de los usuarios del paquete senanal compra los contenidos, pero existe una minoría que obtiene la información por medio de la interacción entre colegas y vecinos, generando así una red social en medio de la comunidad. También fue posible detectar, por medio de entrevistas realizadas, que la movilidad de los distribuidores del PS se realiza en transportes de la red pública y en pocas ocasiones en transportes privados, o sea, el distribuidor viaja a las provincias en ómnibus interprovinciales.

Es posible afirmar que los principales rasgos culturales de la muestra seleccionada es que son personas de naturaleza sociable que forman con los vecinos una gran familia. Una comunidad de muchos negocios, conocida como la más comercial de la ciudad, aunque es uno de los barrios menos desarrollados. El Condado se caracteriza por una amplia cultura popular, con un área de ensayo para carnavales y dos grupos de danza que representan a la comunidad todos los años en competencias de carnaval. La religión más practicada es la "Santería”, proveniente de la cultura africana y conservada por tradición. La lengua hablada en la población es el español como en todo el país, sólo que los condadenses suelen hablar por medio de jergas, a veces difíciles de entender para quien no se encuentra en ese contexto.

De igual forma, fueron elegidos cinco vendedores del PS, también por medio de la observación. Estos vendedores fueron entrevistados enpleando el método de entrevistas en profundidad. Como resultado, fue posible conocer otros actores involucrados en el paquete senanal, como se describe a continuación:

1. Creadores del Paquete Semanal: personas que realizan el compendio de informaciones que integran el paquete senanal, descargan los contenidos digitales y los separan en carpetas genéricas. Estas personas radican en la capital del país y distribuyen el paquete a todas las provincias.

2. Distribuidores 1: son los mienbros de la matriz que distribuyen el paquete senanal en la Habana.

3. Distribuidores 2: distribuyen el PS por todas las provincias de Cuba.

4. Recibidor: recibe el PS al llegar a cada provincia y lleva para los paqueteros. En el caso de esta investigación, son tres paqueteros en la ciudad de Santa Clara.

5. Paqueteros: modifican el paquete senanal de acuerdo con los elenentos identitarios de la comunidad y venden a los vendedores.

6. Vendedores (llenadores de menorias): se sitúan en la base, en las comunidades, venden para los clientes/usuarios, tanto el paquete entero, como contenidos digitales separados.

7. Clientes/usuarios: personas que consumen las informaciones provenientes del PS. 
Laidentificación de los principales actores del paquete semanal permitióla comprensión del papel de cada actor involucrado en el proceso, así como el flujo de circulación para el estudio de caso en la ciudad estudiada. Este flujo de circulación ayudó a entender la red de venta del PS y de los contenidos digitales, pues devido a que es una red comercial los propios mienbros de la cadena actúan como clientes y como proveedores, simultáneamente. Así, los criadores, al ser los primeros en la red comercial, actúan sólo como proveedores. Ya los recibidores, paqueteros y vendedores, actúan no sólo como proveedores, sino también como clientes. En la Figura 3, que sigue a continuación, se muestra el flujo de circulación del paquete semanal.

Figura 3 - Flujo de circulación del Paquete Senanal

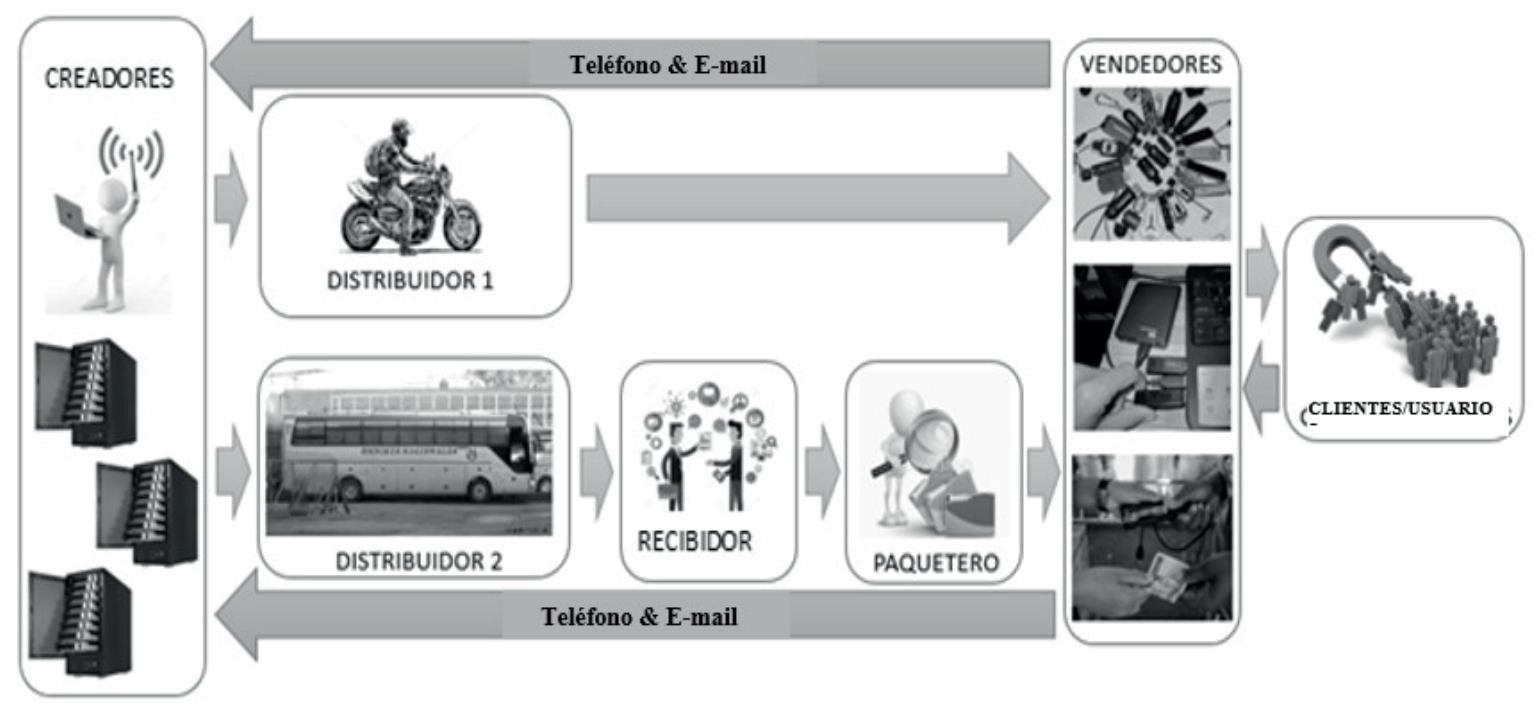

Fuente: Elaboración propia, jun. 2018.

En el flujo de circulación del paquete senanal, están expuestas líneas en dos direcciones opuestas, el canal azul es la ruta de distribución del PS, de los creadores a los clientes/ usuarios. Las flechas verdes indican la retroalimentación de las informaciones, según las solicitudes de los clientes/usuarios. Los canales de comunicación para la retroalimentación pueden ocurrir en el proceso comunicacional de individuo a individuo, o por teléfonos y e-mail. Tenenos como primer elenento, los creadores que repasan el paquete semanal a distribuidores de dos niveles, el distribuidor 1 (motociclista) que reparte el PS directo a los vendedores de la ciudad de La Habana (Cuba) y estos a sus clientes. Mientras el distribuidor 2, lleva el paquete hasta las provincias, donde se encuentran los recibidores, que a su vez entregan a los paqueteros, (tres en el caso de Santa Clara) hasta llegar al destino final: clientes y usuarios. 
Con el mapeo de los actores del enprendimiento, todos los grupos de interés y stakeholders involucrados en el proceso de concepción y comercialización fueron identificados, como participantes directos: i) creadores, ii) distribuidores, iii) recibidores y iv) los llamados paqueteros. Como actores indirectos en la producción del PS, se identificaron: i) TICs, ii) comunidad y sociedad, iii) entidades gubernamentales, iv) instituciones políticas y v) enpresas públicas. Todas estas informaciones fueron analizadas de forma cualitativa en la investigación realizada. Se ilustra, en la Figura 4, el mapa de stakeholders.

Figura 4 - Mapa de Stakeholders

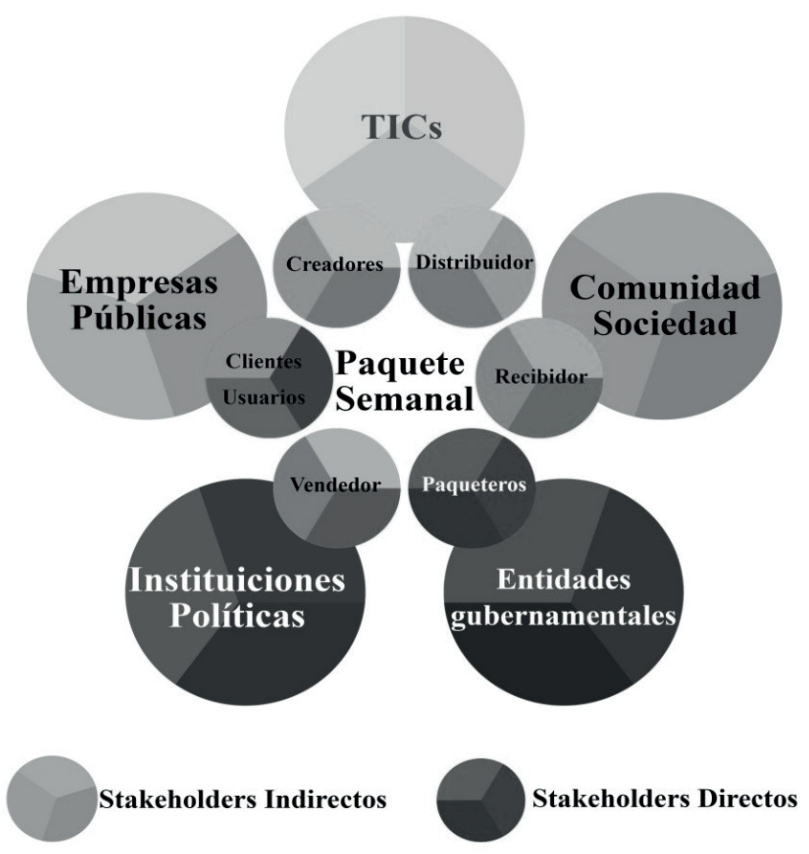

Fuente: Elaboración propia, jun. 2018.

El mapa de stakeholders representa visualmente los grupos de interés. Describe tres niveles: el paquete semanal, los agentes directos y los agentes indirectos. Ambas partes interesadas, directas e indirectas, interactúan entre sí durante todo el sistena de producción, circulación y consumo de este medio alternativo. Así, con la identificación de los grupos de interés realizada y teniendo en cuenta las características culturales de la muestra seleccionada, fueron interpretados los resultados arrojados en los cuestionarios y realizado el análisis cuantitativo univariado.

La primera cuestión tuvo como objetivo conocer la necesidad de uso del Paquete Senanal por la población cubana. En el Gráfico 1, se muestra el comportamiento de la variable necesidad de uso. 
Gráfico 1- ¿Es necesario el paquete senanal para la población cubana?

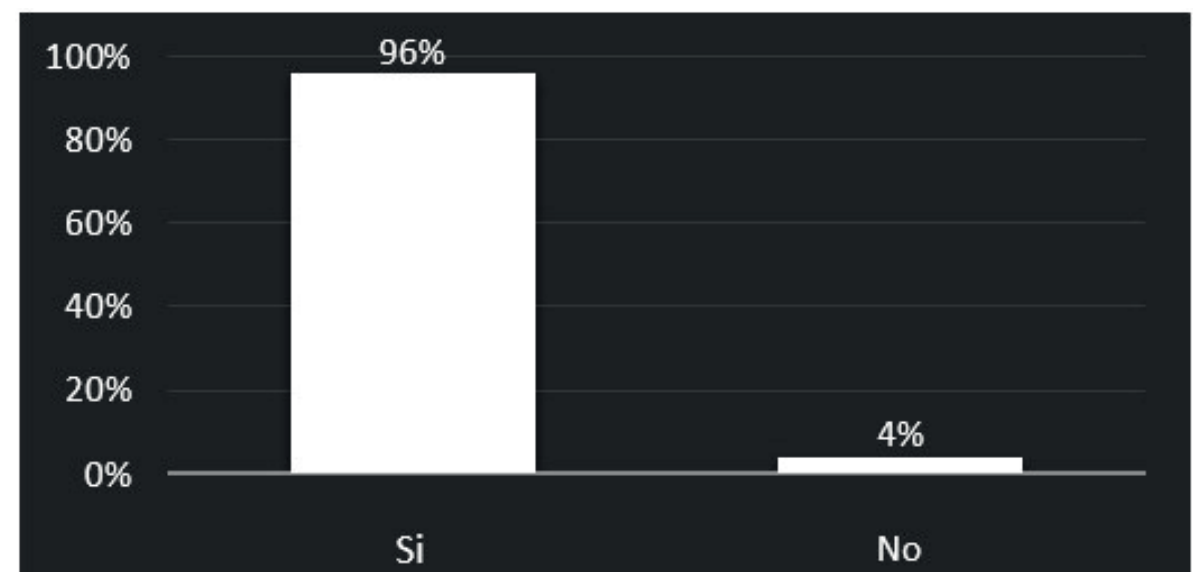

Fuente: Elaboración propia, jun. 2018.

Prácticamente todos los entrevistados consideran el paquete senanal necesario para la población cubana. Sólo dos encuestados discrepan de tal afirmación, lo que equivale al $4 \%$ de la muestra recogida. En contraposición, las 48 personas que consideran importante argumentaron que necesitan el paquete porque es la vía más fácil para adquirir las informaciones que se encuentran em Internet. Tal información permite definir que el PS ocupa un espacio importante en la cotidianidad de la población cubana.

Con el fin de identificar las elecciones informativas de los consumidores, fue elaborada una pregunta sobre las preferencias de consumo del PS, la cual permitió a los entrevistados seleccionar más de una opción, considerando algunos contenidos digitales insertados en el paquete, a saber: 1) entretenimientos; 2) aplicaciones informáticas; 3) documentales; 4) antivirus; 5) deportes; 6) revolico ; 7) software; 8) revistas.

En el Gráfico 2, se puede observar cómo fue la manifestación de las respuestas. Denostrando que casi la totalidad de los encuestados (96\%) afirman que una de las principales informaciones del paquete y sus preferencias son el entretenimiento. Las aplicaciones informáticas ocupan el 60\% de uso; los documentales, el 58\%; y los antivirus, el 54\%, destacándose también entre los ítens de mayor preferencia.

6 Es un sitio de anuncios, que se usa para comercializar, promocionar negocios, permutar casas etc. 
Gráfico 2 - Preferencias de contenidos digitales

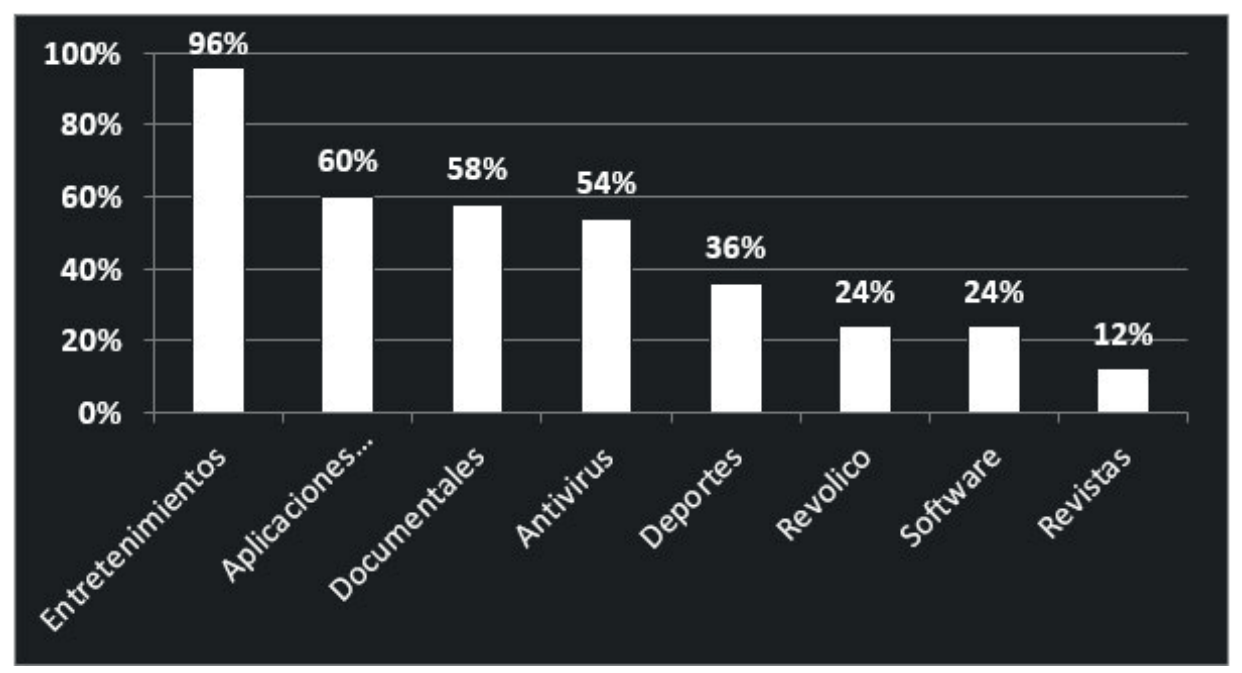

Fuente: Elaboración propia, jun. 2018.

Debido al alto porcentaje de los usuarios que prefieren el entretenimiento, 48 de los 50 encuestados prefieren el contenido audiovisual, como novelas, series, películas y reality shows. Una cuestión que trató de la búsqueda sobre el principal modo de obtener la información de los contenidos digitales del PS fue incluida en el cuestionario y presenta sus resultados a continuación, de acuerdo con el Gráfico 3.

Gráfico 3 - Modo de obtención de los contenidos digitales

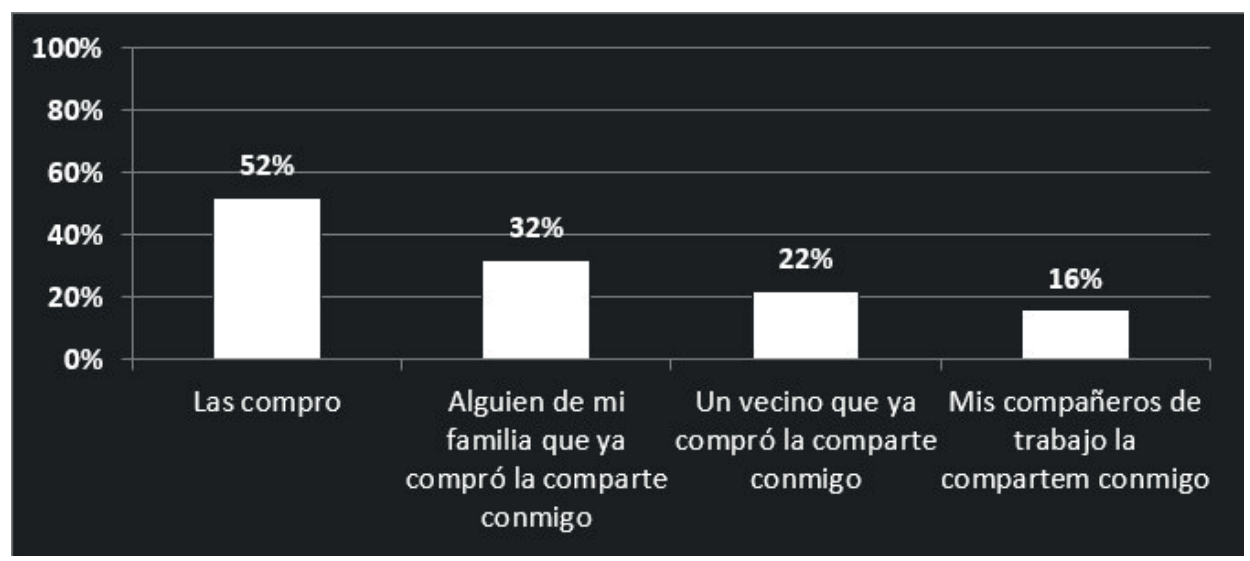

Fuente: Elaboración propia, jun. 2018.

Se constató que el 52\% de los encuestados adquieren las informaciones que necesitan por medio de la compra. Sin enbargo, el 70\% las reciben por medio de compartimiento, detallado en un 32\% que obtiene las informaciones por alguien de la familia que realizó la 
compra, el 22\% de algún vecino que compró y el 16\% alegó recibir las informaciones por parte de sus compañeros de trabajo. Se deduce que este método de compartir los contenidos digitales entre los consumidores del PS genera la interacción y establece una red social entre la población. Los usuarios fueron encuestados también, sobre el tienpo de consumo de las informaciones del PS, y el comportamiento de estas respuestas se reflejan en el Gráfico 4.

Gráfico 4 - Tienpo de consumo del PS



Fuente: Elaboración propia, jun. 2018.

Como se indica en el Gráfico 4, todos los encuestados afirman utilizar los contenidos digitales al menos una vez al mes. Siendo así, el 46\% afirma utilizar el PS todos los días, el 24\% accede en días alternos, restando sólo el 6\% que dijeron utilizar una vez al mes. La mayoría de los entrevistados, por lo tanto, accede al menos una vez por senana, representando el 94\% de la muestra seleccionada. El alto porcentaje de acceso a la PS muestra que el consumo de los contenidos digitales se realiza con frecuencia regular. Entre las preferencias por televisión nacional o audiovisual del PS, el resultado se muestra en el Gráfico 5.

Gráfico 5 - Preferencias de audiovisuales entre latelevisión y el PS



Fuente: Elaboración propia, jun. 2018. 
Los programas del paquete semanal tienen una amplia ventaja de preferencia de los entrevistados cuando se comparan con los programas de la televisión nacional, ya que el 94\% asegura preferir los contenidos del paquete, sólo el 6\% opta por la televisión nacional. La oferta y la calidad de los servicios de programación de audiovisuales en la televisión nacional no cumplen las expectativas de la población, por lo que los individuos buscan en el paquete lo que no logran por vías formales. En cuanto al servicio prestado, se observa que sólo el 2\% de los encuestados dijo que no estaban satisfechos. En contraposición, el $80 \%$ afirma estar satisfechos con la atención de los vendedores, conforme presentado en el Gráfico 6.

Gráfico 6 - Satisfacción del servicio recibido

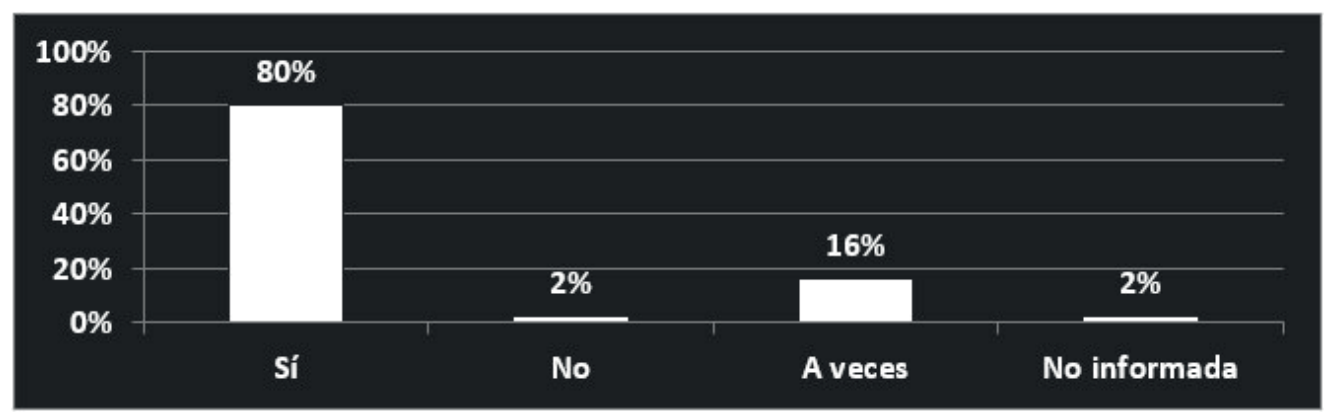

Fuente: Elaboración propia, jun. 2018.

De acuerdo con los datos obtenidos, los vendedores del PS enplean estrategias de diseño de servicios para ganar y preservar a los clientes. En cuanto a la comprensión del funcionamiento del proceso de ventas del paquete, se indagó sobre la selección de los proveedores del PS y la elección de los usuarios, conjuntamente, se buscaron observar las características de los servicios prestados por los vendedores. De esta forma, fueron combinados elenentos positivos y negativos, determinados en nueve alternativas para ser elegidas, ella son: 1) Falta de educación en el saludo; 2) Carácter amable y buena atención; 3) No me venden la información que deseo; 4) Rapidez en el servicio; 5) Poco conocimiento de la información que estoy comprando; 6) Sugestiones de las informaciones que pueden interesarme; 7) Maltrato en la relación interpersonal; 8) Equivocación al devolver la menoria flash; 9) Denora al venderme la información.

Además de estas expresiones del servicio, los entrevistados tuvieron la posibilidad de sugerir outras que no se encontraron entre las presentadas. El Gráfico 7 indica el resultado de esta interrogante. 
Gráfico 7- Características del servicio



Fuente: Elaboración propia, jun. 2018.

Tres opciones se destacaron entre las características de la prestación de servicio encontradas en la compra del paquete senanal: carácter amable y buena atención (72\%), rapidez en el servicio (64\%) y sugerencias de las informaciones que pueden generar interés (52\%). Los valores no totalizan el 100\% porque se permite que el entrevistado indique más de una característica. Se observa que, de nueve variantes dadas, tres de ellas eran positivas y el resto, negativas. No obstante, los mayores porcentajes estuvieron en los puntos favorables del servicio. Por consiguiente, y teniendo en cuenta las respuestas de las cuestiones anteriores, puede decirse que los vendedores del PS se preocupan en garantizar que las experiencias de los clientes al interactuar con el servicio sean agradables y satisfactorias. En relación con el costo-beneficio de las informaciones del PS, los encuestados opinaron, según se presenta en el Gráfico 8.

Gráfico 8 - Relación costo-beneficio



Fuente: Elaboración propia, jun. 2018. 
Se observa que el $78 \%$ de los encuestados considera los precios de venta asequibles, teniendo en cuenta el costo-beneficio, el $14 \%$ juzga caro, el $4 \%$ considera barato y el $4 \%$ prefirió no responder a la pregunta, lo que representa a dos personas. Las respuestas obtenidas permiten definir que los clientes/usuarios del PS consideran justos los precios que pagan por las informaciones que necesitan, lo que explica que busquen tales contenidos informativos en el paquete y no accediendo a Internet. Por último, considerando el carácter holístico del servicio establecido entre los vendedores del PS como proveedores y los clientes usuarios, se investigó la cuestión sobre la solicitud de información por parte de los usuarios a sus proveedores. El Gráfico 9 presenta los resultados correspondientes a la pregunta.

Gráfico 9 - Solicitación de información

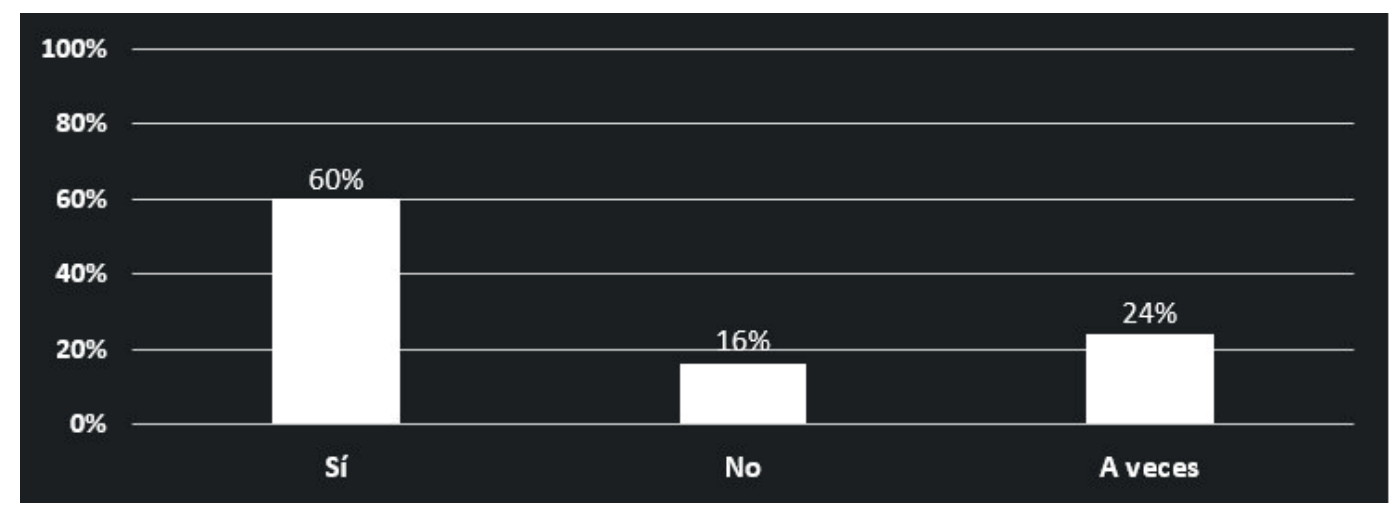

Fuente: Elaboración propia, jun. 2018.

Se entiende que el $84 \%$ de los encuestados afirman solicitar a su proveedor cuando no encuentran alguna información en el paquete senanal. Estos datos revelan que el $60 \%$ de los usuarios piden la información que desean, el 24\% dice que sólo lo hacen algunas veces y el $16 \%$ afirma que no solicitan información. De esta forma, el servicio generado por la comercialización y venta del PS posee una forma holística, compuesta por las necesidades y preferencias informacionales de los clientes.

\section{Consideraciones finales}

El uso de las TICs representa una variación considerable en la sociedad, un cambio en la educación, las relaciones interpersonales y la forma de difundir y generar conocimientos. El desarrollo tecnológico es una prioridad en la comunicación hoy en día y la limitación de acceso representa una importante diferencia entre los países desarrollados y en vías de desarrollo.

Las limitaciones de acceso a Internet en Cuba impulsaron a la sociedad cubana a buscar vías y soluciones para sus necesidades informativas y comunicacionales, haciendo que surjan modos alternativos de tecnologías y, con ellas, nuevos negocios. Adenás de forzar el 
enprendedorismo, las startups y las personas sin formación en diseño aplican, intuitivamente, herramientas atribuidas al diseño de servicios en la creación, comercialización y venta de este producto digital, que es el PS. La distribución y circulación de este paquete senanal en Cuba han creado espacio para la interacción y el intercambio de informaciones entre las personas, adenás de proporcionar unanueva forma de ingresos monetarios en la sociedad. Esta situación posibilitó la ocurrencia de la modificación de la cultura, confrontando tradiciones y alterando estilos de vida, costumbres, gustos, intereses, lenguaje, entre outras.

Es posible afirmar que las lagunas existentes en la programación oficial de la televisión cubana imponen el surgimiento de outras formas de adquirir información. La falta de acceso a Internet también acarrea lagunas en diversos sentidos por parte de la población estudiada, convirtiéndose así el PS en un fuerte competidor de la enpresa de telecomunicaciones, que es responsable del funcionamiento de Internet en el país. El PS como negocio y generador de servicios enplea las herramientas del diseño de servicios, pues es un producto enfocado en el usuario y que toma en consideración la forma como el mismo va a consumir o interactuar con la información, adenás de buscar la satisfacción de sus necesidades y los deseos, solucionando la carencia informacional de manera eficiente y eficaz.

El PS permite al usuario acceder y pasar las informaciones con relativa facilidad, estableciendo intercambios con vecinos, amigos y compañeros de trabajo y/o compañeros de escuela o, incluso, enplear una de las vías aquí presentadas para obtenerlo. Para la determinación de los contenidos digitales del paquete senanal, los creadores utilizan como herramientas la co-creación y el diseño de servicios, en la conversión de un negocio popular, como forma de pensar en la mejor oferta de servicios y en la garantía de la buena calidad del producto, lo que por sí, ya representa un reflejo de la construcción social de este fenómeno.

\section{Referencias}

BANCO CENTRAL DE CUBA. Tipo de Cambio. Disponible en: http://www.bc.gob.cu/espanol/tipo_ cambio.asp. Acceso en: 9 abr. 2016.

BAnCO METROPOlTANO DE CUBA. Tipo de Cambio Banco Metropolitano. Disponible en: http:// www.bc.gob.cu/espanol/tipo_cambio_METROPOLITANO.asp. Acceso en: 9 abr. 2016.

CONCEPCIÓN, J. R. Escaneando el Paquete Senanal (I) (+ Infografía). Disponible en: http://www. cubadebate.cu/noticias/2015/10/19/el-paquete-senanal-i-infografia/\#.WCFdR9LhDIU. Acceso en: 19 abr. 2016a.

CONCEPCIÓN, J. R. Escaneando el Paquete Senanal (II). Disponible en: http://www.cubadebate.cu/tenas/ cultura-tenas/2015/10/20/el-paquete-senanal-ii/. Acceso en: 19 abr. 2016b.

CONSEJO POPULAR. EcuRed. Disponible en: https://www.ecured.cu/Consejo_Popular. Acceso en: 17 nov. 2017.

CONSTITUCIÓN DE LA REPÚBLICA DE CUBA. Constitución de la República de Cuba, 1976. Disponible en: http://www.cuba.cu/gobierno/cuba.htm. Acceso en: 17 out. 2016.

ETECSA. EcuRed. Disponible en: https://www.ecured.cu/ETECSA. Acceso en: 20 out. 2016. 
MARANHÃO, A. C. K. O jornalista brasileiro: convergência e mudança provocada pelas Tecnologias da Informação e Comunicação.1. ed. Brasília: Editora Universidade de Brasília; FAC Livros, 2017.

MINCOM. Telecomunicaciones. Disponible en: http://www.mincom.gob.cu/?q=telecomunicaciones. Acceso en: 19 fev. 2018.

MINISTERIO DE JUSTICIA. Resolución No. 11/05 del Ministerio de Trabajo y Seguridad Social. Gaceta Oficial de la República de Cuba, v. 12, p. 64-65, 2005. Disponiblel en: http//legislacion.sld.cu/index. php?P=DownloadFile\&Id=501. Acceso en: 19 fev. 2018.

PINHEIRO, T. D. The Service Startup. Inovação e Enpreendedorismo através do Design Thinking. 1ra. ed. Rio de Janeiro - RJ: [s.n.], 2015.

SAMPIERI, R. H.; COLLADO, C. F.; LUCIO, P. B. Metodologia de la investigacion. 5ta Edicio ed. México: [s.n.], 2014. Disponible en: http://www.acadenia.edu/6399195/Metodologia_de_la_investigacion_5ta_ Edicion_Sampieri. Acceso en: 19 fev. 2018.

STICKDORN, M.; SCHNEIDER, J. Isto é Design Thinking de Serviços. Fundamentos-FerramentasCasos. Porto Alegre: [s.n.], 2014.

STICKDORN, M.; SCHNEIDER, J. This is service design thinking. Basics, Tools, Cases. Amsterdam: [s.n.], 2011.

\section{Yanet Castellanos Argüelles}

Graduada en Comunicación Social por la Universidad Central Marta Abreu de Las Villas, Cuba (2008), técnica en Trabajo Social por la Escuela Formadora de Trabajadores Sociales de Villa Clara (2002) en curso conveniado con la universidad anterior, y Máster en Diseño por el Programa de Pos graduación de Diseño del Instituto de Artes de la Universidad de Brasilia (2018). Posee experiencia en el área de Trabajo Social y Comunicación e Información. En Cuba, trabajó en varios proyectos comunitarios en diferentes áreas de la provincia Villa Clara, y algunos de estos enfocados en el autodesarrollo comunitario, impulsando procesos informacionales y comunicacionales en la comunidad. E-mail: yanetca00@gmail.com.

\section{Ana Carolina Kalume Maranhão}

Doctora en Comunicación por la Universidad de Brasilia (2014), Máster en Comunicación por la Universidad de Brasilia (2008). Realizó especialización em Bioética por la Cátedra Unesco de Bioética de la Universidad de Brasilia (2004). Es profesora de la Facultad de Comunicación, de la Universidad de Brasilia y profesora colaboradora del Programa de Posgraduación en Diseño de la Universidad de Brasilia. Desarrolla investigaciones sobre Comunicación, Redes, Tecnologías de la Información y Comunicación y Educación, a partir de nuevas metodologías de aprendizaje. Actúa en el área de Comunicación, con énfasis en Producción de Contenido, Estrategias y Procesos Comunicacionales, Sistenas de Comunicación, Educación y PBL. E-mail: ckalume@gmail.com. 


\section{Rogerio José Camara}

Doctor y Máster en Comunicación por la Universidad Federal de Rio de Janeiro, graduado en Comunicación Visual por la Pontificia Universidad Católica de Rio de Janeiro. Profesor de la Universidad de Brasilia. Posee experiencia profesional en las áreas de Diseño y Educación. Participa como docente del programa de posgraduación en Artes y del PPG Design, ambos de la Universidad de Brasilia. Realiza investigaciones sobre las relaciones entre escrita y ciudad con énfasis en la poesía visual yen las nuevas tecnologías. E-mail: rogeriojcamara@gmail.com.

Recibido en: 10.10.2018

Aceptado en: 28.05.2019 